\section{Do Chloroquine and Hydroxychloroquine Treat COVID-19?}

\section{Abstract}

Chloroquine is an anti-malarial and autoimmune disease drug, which has an antiviral activity. In vitro, the Chloroquine and Hydroxychloroquine are potent at inhibiting SARS-CoV. Therefore, Chloroquine and Hydroxychloroquine are being studied for their potential treatment of coronavirus disease 2019. Those drugs are now recommended by the FDA, and multiple health organizations. This aims of this review is to explore the efficacy of the chloroquine and hydrochloroquine in the treatment of the novel COVID-19.

Keywords: COVID-19; Chloroquine; Hydroxychloroquine

\section{Bouhmou Ayoub*, Bzikha Reda, Bzikha Ilham and Bouchnafati Sanaa}

Faculty of Medicine and Pharmacy, University of Sidi Mohamed Ben Abdellah, Fes, Morocco

\author{
*Corresponding author: Bouhmou Ayoub \\ ayoub_1900@hotmail.fr; \\ redabzikha@gmail.com
}

Tel: 0771459523

Faculty of Medicine and Pharmacy, University of Sidi Mohamed Ben Abdellah, Fes, Morocco.

Citation: Ayoub B, Reda B, Ilham B, Sanaa B (2020) Do Chloroquine and Hydroxychloroquine Treat COVID-19?. Arch Med Vol. 12 No.6:35

COVID-19 on April 1, 2020 [12].

And the sixth edition chloroquine recommended chloroquine phosphate tablet, $500 \mathrm{mg}$ twice per day for 10 days for patients diagnosed as mild, moderate and severe cases of novel coronavirus pneumonia and without contraindications to chloroquine and in the Seventh Edition of the Guidance, issued on March 3, 2020, recommends a reduced dosage and shortened duration of treatment to reduce the risk of adverse effects of chloroquine [13].

The dosage of chloroquine phosphate for adult patients with a body weight greater than $50 \mathrm{~kg}$ is $500 \mathrm{mg}$ twice a day for 7 days and that for adult patients with a body weight less than $50 \mathrm{~kg}$ is $500 \mathrm{mg}$ twice a day for the first 2 days and $500 \mathrm{mg}$ once a day for the following 3-7 days [13].

\section{Results and Discussion}

However, in vitro anti-SARS-CoV-2 finding Hydroxychloroquine superior to Chloroquine $[5,6]$ recommending Hydroxychloroquine sulfate $400 \mathrm{mg}$ twice daily on day 1, followed by $200 \mathrm{mg}$ twice daily for the next 4 days to treat COVID- 19 [14] as it has been shown by in vitro results were reported by the Wuhan Institute of Virology [5]. And we report that Gautret, et al. were the first 
to report promising in vivo data of Hydroxychloroquine in a nonrandomized clinical trial [5].

Furthermore, the administration of hydroxychloroquine $200 \mathrm{mg}$, three times a day led to a significant reduction in viral carriage on day 6 post-treatment compared to that in the control group, which did not receive hydroxychloroquine, adding azithromycin to hydroxychloroquine appears to more efficiently clear the virus, treatment with hydroxychloroquine and azithromycin resulted in a rapid decline in the nasopharyngeal viral load [15].

Another study showed that while hydroxychloroquine is administered orally in a loading dose of $400 \mathrm{mg}$ twice daily, followed by a maintenance dose of $200 \mathrm{mg}$ twice daily for four days, leads to a significant reduction of viral load and respiratory symptoms [14].

Clinical study showed that hydroxychloroquine helped reduce the time until body temperature returned to normal, it decreased the duration of cough, and it improved lung imaging findings COVID-19 [16] and it has been recommended the use of Hydroxychloroquine as a possible prophylaxis and curative treatment for COVID-19

\section{References}

1 Colson P, Rolain JM, Raoult D (2020) Chloroquine for the 2019 novel coronavirus SARS-CoV-2. Int J Antimicrob Agents 55: 105923.

2 Colson P, Rolain JM, Lagier JC, Brouqui P, Raoult D (2020) Chloroquine and hydroxychloroquine as available weapons to fight COVID-19. Int J Antimicrob Agents 55: 105932.

3 Vincent MJ, Bergeron E, Benjannet S, Erickson BR, Rollin PE, et al. (2005) Chloroquine is a potent inhibitor of SARS coronavirus infection and spread. Virol J 2: 69

4 Wang M, Cao R, Zhang L, Yang X, Liu J, et al. (2020) Remdesivir and chloroquine effectively inhibit the recently emerged novel coronavirus (2019-nCoV) in vitro. Cell Res 30: 269-271.

5 Liu J, Cao R, Xu M, Wang X, Zhang H, et al. (2020) Hydroxychloroquine, a less toxic derivative of chloroquine, is effective in inhibiting SARSCoV-2 infection in vitro. Cell Discov 6: 16.

6 Biot C, Daher W, Chavain N, Fandeur T, Khalife J, et al. (2006) Design and synthesis of hydroxyferroquine derivatives with antimalarial and antiviral activities. J Med Chem 49: 2845-2849.

7 The Multicenter Collaboration Group (2020) Department of Science and Technology of Guangdong Province and Health Commission of Guangdong Province for Chloroquine in the Treatment of Novel Coronavirus Pneumonia: Expert consensus on chloroquine phosphate for the treatment of novel coronavirus pneumonia Zhonghua Jie He He Hu Xi Za Zhi 43: 185-188.

8 Gao J, Tian Z, Yang X (2020) Breakthrough: Chloroquine phosphate has shown apparent efficacy in treatment of COVID-19 associated pneumonia in clinical studies. Biosci Trends 14: 72-73.

9 http: //en.nhc.gov.cn/

10 https: //e.dxy.cn/broadcast/live/id/13818?source=pagesidebar

11 Lenzer J (2020) COVID-19: US gives emergency approval to hydroxychloroquine despite lack of evidence. BMJ 369: m1335.

12 https: //www.ema.europa.eu/en/news/COVID-19-chloroquine-
$[1,2]$. Besides, azithromycin has been found to have antiviral and anti-inflammatory effects in both in vivo and in vitro studies and also it has activity against pro-inflammatory cytokines $[17,18]$.

Azithromycin combined to hydroxychloroquine result numerically superior viral clearance compared with hydroxychloroquine monotherapy [17]. Thus, Chloroquine and hydroxychloroquine can cause rare and serious adverse effects, including QTc prolongation ventricular arrhythmias, especially when prescribed with azithromycin hypoglycemia, neuro psychiatric effects fulminant hepatic failure, and retinopathy serious cutaneous adverse reactions [19-23].

\section{Conclusion}

However, Hydroxychloroquine, a more tolerable derivative of chloroquine, also displayed potent activity against SARS-CoV-2 in vitro. Finally, we suggest further investigation in large scale studies, but, as those drugs show efficacy in vitro and in vivo and we may use them as treatment of COVID-19 until the discovery of a vaccine.

hydroxychloroquine-only-be-used-clinical-trials-emergency-useprogrammes

13 Guidance for COVID-19: Prevention, Control, Diagnosis, and Management. National Health Commission. 6 Edn.

14 Yao X, Ye F, Zhang M, Cui C, Huang B, et al. (2020) In Vitro Antiviral Activity and Projection of Optimized Dosing Design of Hydroxychloroquine for the Treatment of Severe Acute Respiratory Syndrome Coronavirus 2 (SARS-CoV-2). Clin Infect Dis 71: 732-739.

15 Gautret P, Lagier JC, Parola P, Hoang VT, Meddeb L, et al. (2018) Hydroxychloroquine and azithromycin as a treatment of COVID-19: results of an open-label non-randomized clinical trial. Int J Antimicrob Agents 56: 105949

16 Chen ZW, Hu JJ, Zhang ZW, Jiang S, Han SM, et al. (2020) Efficacy of hydroxychloroquine in patients with COVID-19: Results of a randomized clinical trial. MEDRXIV.

17 Amsden GW (2005) Anti-inflammatory effects of macrolides - An underappreciated benefit in the treatment of community-acquired respiratory tract infections and chronic inflammatory pulmonary conditions? J Antimicrob Chemother 55: 10-21.

18 Gielen V, Johnston SL, Edwards MR (2010) Azithromycin induces antiviral responses in bronchial epithelial cells. Eur Respir J 36: 646-654.

19 Kalil AC (2020) Treating COVID-19-off-label drug use, compassionate use, and randomized clinical trials during pandemics. JAMA 323 1897-1898.

20 Juurlink D (2020) Coronavirus (COVID-19) update: Chloroquine/ hydroxychloroquine and azithromycin. JAMA

21 Murphy M, Carmichael AJ (2001) Fatal toxic epidermal necrolysis associated with hydroxychloroquine. Clin Exp Dermatol 26: 457-458.

22 Makin AJ, Wendon J, Fitt S, Portmann BC, Williams R (1994) Fulminant hepatic failure secondary to hydroxychloroquine. Gut 35: 569-570.

23 Chorin E, Dai M, Shulman E, Wadhwani L, Cohen BR, et al. (2020) The QT interval in patients with SARS-CoV-2 infection treated with hydroxychloroquine/azithromycin. MEDRXIV. 